\title{
The Effect of Mechanical Vibration Stimulation of Perception Subthreshold on the Muscle Force and Muscle Reaction Time of Lower Leg
}

\author{
Huigyun Kim, ${ }^{1}$ Kiyoung Kwak, ${ }^{1}$ and Dongwook Kim ${ }^{2,3}$ \\ ${ }^{1}$ Department of Healthcare Engineering, Graduate School, Chonbuk National University, Jeonju 54896, Republic of Korea \\ ${ }^{2}$ Department of Biomedical Engineering, College of Engineering, Chonbuk National University, Jeonju 54896, Republic of Korea \\ ${ }^{3}$ Research Center of Healthcare and Welfare Instrument for the Aged, Chonbuk National University, Jeonju 54896, Republic of Korea \\ Correspondence should be addressed to Dongwook Kim; biomed@jbnu.ac.kr
}

Received 15 January 2016; Accepted 10 May 2016

Academic Editor: Justin Keogh

Copyright (C) 2016 Huigyun Kim et al. This is an open access article distributed under the Creative Commons Attribution License, which permits unrestricted use, distribution, and reproduction in any medium, provided the original work is properly cited.

\begin{abstract}
The objective of this study is to investigate the effect of mechanical vibration stimulation on the muscle force and muscle reaction time of lower leg according to perception threshold and vibration frequency. A vibration stimulation with perception threshold intensity was applied on the Achilles tendon and tibialis anterior tendon. EMG measurement and analysis system were used to analyze the change of muscle force and muscle reaction time according to perception threshold and vibration frequency. A rootmean-square (RMS) value was extracted using analysis software and Maximum Voluntary Contraction (MVC) and Premotor Time (PMT) were analyzed. The measurement results showed that perception threshold was different from application sites of vibration frequency. Also, the muscle force and muscle reaction time showed difference according to the presence of vibration, frequency, and intensity. This result means that the vibration stimulation causes the change on the muscle force and muscle reaction time and affects the muscles of lower leg by the characteristics of vibration stimulation.
\end{abstract}

\section{Introduction}

Decreased balance and muscle force with aging cause unstable gait, which often results in falls [1]. Gait is a continuous and repeated movement requiring ability to balance [2]. The ability to balance for gait requires integrated actions of sensory organs, central nerve system, ability to manage exercise, and muscle functions [3]. However, older adults have decreased speed of transferring signals in central nerve system as a response to stimuli for maintaining balance. And weakened muscle force of lower limb decreases the speed, stride, and step frequency and as a result, the risk of falls increases [4]. To prevent falls of the older adults, many studies have been conducted on the improvement of balance and strengthening of muscle force.

Somatosensory vibration stimulation has been proven to decrease the body sway and to increase the postural stability by some studies [5-9]. Eklund reported that the activation of muscles using vibration helped the stability of posture [10].
Also there are some studies that investigated the effect of vibration stimulation on the muscle functions. Curry and Clelland reported that the vibration stimulation increased MVC (Maximum Voluntary Contraction) [11], and Kang et al. reported that the systemic vibration exercise stimulated muscle nerves and shortened the muscle reaction time of the muscles [12].

However, many previous studies used only vibration stimulus with single characteristic and the different characteristics of individual vibration were not taken into consideration. Moreover, Pacinian corpuscle, a sensory receptor responding to vibration stimulation, was reported to respond sensitively at the frequency of $100 \mathrm{~Hz}-300 \mathrm{~Hz}$ [13-15]. However, such frequency range of vibration stimulation was too broad to investigate its effect on the muscle activities. As a result, a previous study was conducted to measure perception threshold to vibration stimulation and to investigate narrower frequency range to which sensory receptors respond more sensitively [16]. From the result of the study, this study will 
analyze the effect of vibration on the muscle force and muscle reaction time of lower leg according to perception threshold using MVC and PMT.

\section{Materials and Methods}

2.1. Subjects. Ten adult males (age: $26.9 \pm 1.5$, height: $171.2 \mathrm{~cm}$ \pm 2 , and weight: $65.9 \mathrm{~kg} \pm 5.3$ ) participated in this study. The subjects did not have any musculoskeletal diseases and neurological diseases. The subjects did not exercise regularly and have not had experienced special body and vibration exercise. The study was approved by the IRB of Chonbuk National University (IRB File number JBNU 2015-06-012).

2.2. Equipment. The small-linear actuator was used to apply vibration. A function generator was used to modify the intensity and frequency of vibration. To fix the small-linear actuator at the Achilles tendon and tibialis anterior tendon, we used rubber band. For EMG measurement, the electrodes (DELSYS Inc., Surface EMG Sensor) were attached on the tibialis anterior muscle and triceps surae and ground electrode was attached on the patella to obtain the muscle force and muscle reaction time. An auditory signal was prepared to measure PMT. The signal and vibration were applied simultaneously.

2.3. Measurement and Extraction of Muscle Force and Muscle Reaction Time. To investigate the change of muscle force and muscle reaction time by applying vibration stimulation, the MVC (Maximum Voluntary Contraction) and PMT (Premotor Time) were measured. For this purpose, EMG measurement system (Bagnoli ${ }^{\mathrm{TM}}$ Desktop EMG Systems, DELSYS Inc., USA) was used. The MVC is the maximum force which a subject can produce voluntary. PMT is the time that takes to reach the motor end plate through the cortex of the cerebrum and motor neuron from the point of stimulation. Therefore, PMT refers to the reaction time by nerve element [17]. The subject performed the MVC at the same time as vibration applied and measured the peak value of RMS (root-mean-square) EMG. The subject performed the muscle contraction at the same time as auditory signal. The time to muscle activation start time from the apply point of the auditory signal was extracted from the RMS EMG (Figure 1).

2.4. Protocol. In order to investigate the change in the muscle force and muscle reaction time in accordance with the characteristics of the vibration stimulation (frequency, intensity), we made the vibration stimulation conditions. Based on previous studies that investigated vibration perceptive threshold according to vibration frequency, $180 \mathrm{~Hz}$, $190 \mathrm{~Hz}$, and $250 \mathrm{~Hz}$ were selected as the vibration stimulation frequency. Likewise, intensity of perception threshold (100\%) and subthreshold (80\%) was selected as the vibration stimulation intensity [16]. Seven vibration conditions were set by combining nonstimulation, frequency of vibration, and intensity of vibration. With MVC and PMT measured 3 times

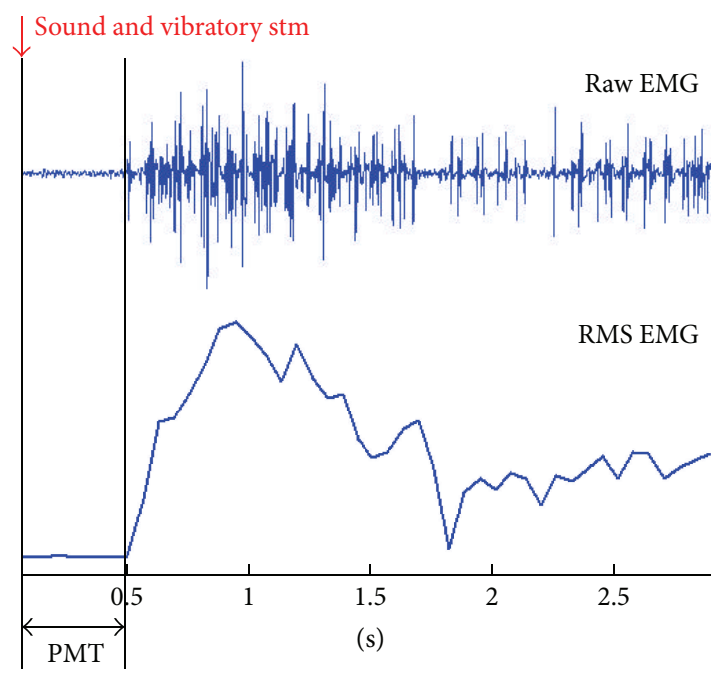

FIgURE 1: PMT extraction example.

in each condition, the stimulation was applied randomly, with 3 minutes of break time after each trial.

2.5. Data Analysis. Based on the MVC value of nonstimulation conditions, increase and decrease of change rate of MVC value of vibration stimulation condition were analyzed. PMT was analyzed by the same method. In this study, we conducted statistical analysis on the result using the Wilcoxon Signed Ranks Test of SPSS 18.0. The result was validated at the significance level of $p<0.05$.

\section{Results}

3.1. Change of Muscle Force according to the Characteristics of Vibration. The following is the results of MVC according to the vibration stimulation applied to the muscles of the lower leg through the Achilles tendon and the tibialis anterior tendon. Table 1 shows the increase rate of muscle force [\%] when the vibration was applied on the Achilles tendon and the tibialis anterior tendon to stimulate triceps surae and tibialis anterior muscle. The increase rate of muscle force [\%] was obtained by comparing with the MVC data without stimulation.

Table 1 showed that the muscle force increased with the vibration stimulation for both the triceps surae and tibialis anterior muscle compared without stimulation. The strength of both triceps surae and tibialis anterior muscle significantly increased with the vibration applied on the Achilles tendon (Soleus $p$ value $=0.022$, Lateral Gastrocnemius $p$ value $=0.049$, Medial Gastrocnemius $p$ value $=$ 0.033 , and tibialis anterior $p$ value $=0.047$ ). The strength of Medial Gastrocnemius muscle significantly increased with the vibration applied on the tibialis anterior tendon (Medial Gastrocnemius $p$ value $=0.017$ ). The strength of Soleus muscle, Lateral Gastrocnemius muscle, and tibialis anterior muscle increased compared to that without stimulation without showing statistical significance (Soleus $p$ value $=$ 
TABLE 1: The increase rate of muscle force by vibration stimulation applied on the Achilles tendon and tibialis anterior tendon [\%].

\begin{tabular}{lcccc}
\hline & Soleus & Lateral Gastrocnemius & Medial Gastrocnemius & Tibialis anterior \\
\hline Achilles tendon & $10.01^{*} \pm 7.03$ & $11.97^{*} \pm 7.12$ & $14.52^{*} \pm 7.99$ & $10.51^{*} \pm 7.02$ \\
Tibialis anterior tendon & $6.53 \pm 5.30$ & $9.83 \pm 6.43$ & $9.34^{*} \pm 5.41$ & $5.74 \pm 5.75$ \\
\hline
\end{tabular}

${ }^{*} p<0.05$. Nonstimulation versus stimulation.

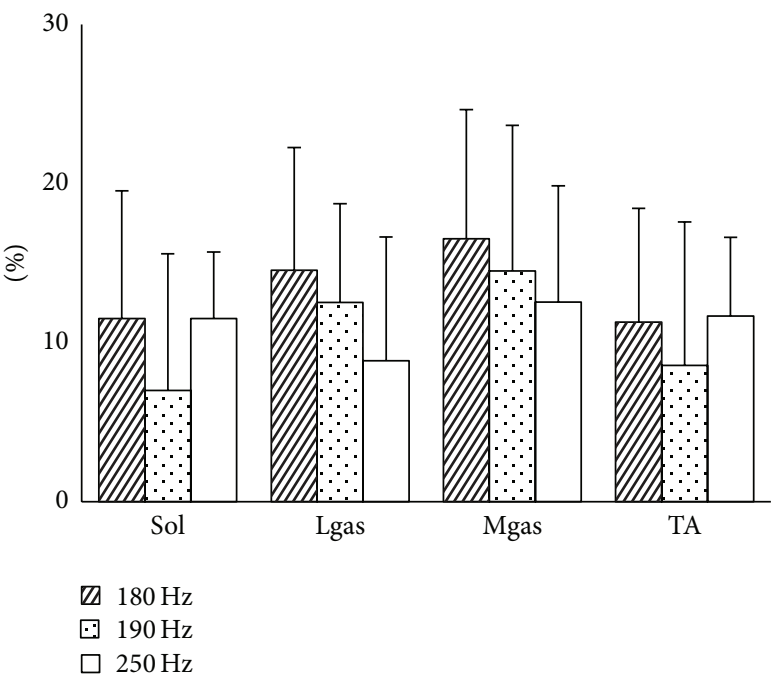

(a)

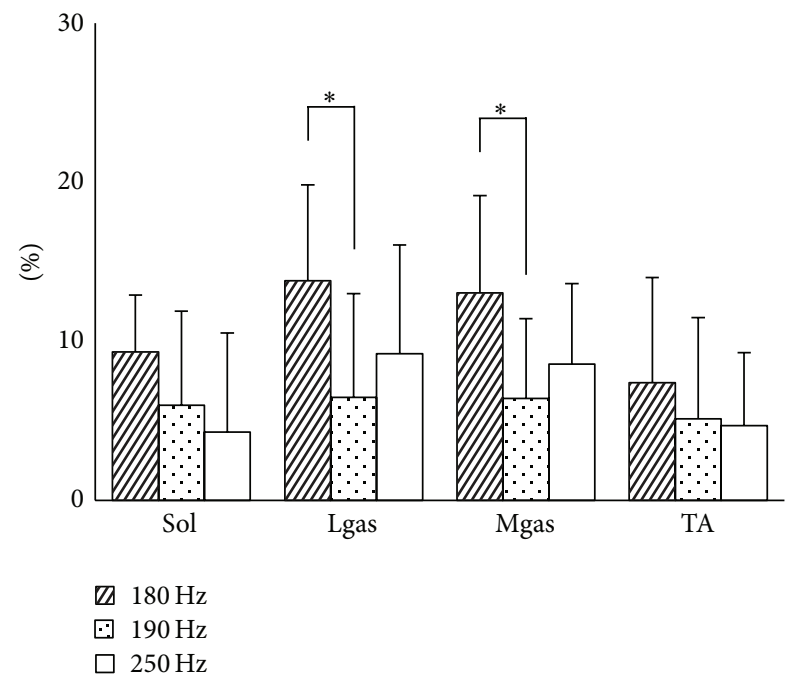

(b)

FIGURE 2: The increase rate of muscle force by the vibration frequency applied on the Achilles tendon and tibialis anterior tendon [\%] $\left({ }^{*} p<\right.$ 0.05. $180 \mathrm{~Hz}$ versus $190 \mathrm{~Hz}$ ). (a) Achilles tendon. (b) Tibialis anterior tendon.

0.678 , Lateral Gastrocnemius $p$ value $=0.102$, and tibialis anterior $p$ value $=0.221$ )

Figures 2(a) and 2(b) show the increase rate of muscle force [\%] by the vibration frequency applied on the Achilles tendon and tibialis anterior tendon.

The increase rate of muscle force was the greatest at $12 \%$ and $11 \%$ with the vibration frequency of $180 \mathrm{~Hz}$ and the smallest at $7 \%$ and $9 \%$ with the frequency of $190 \mathrm{~Hz}$ when the vibration was applied on the Soleus muscle and tibialis anterior muscle, respectively (Figure 2(a)). The increase rate of muscle force was the greatest at $15 \%$ and $17 \%$ with the vibration frequency of $180 \mathrm{~Hz}$ and the smallest at $9 \%$ and $13 \%$ with the frequency of $250 \mathrm{~Hz}$ when the vibration was applied on the Lateral Gastrocnemius muscle and Medial Gastrocnemius muscle, respectively. (Figure 2(b)) The increase rate of muscle force was the greatest at 9 and $7 \%$ with the vibration frequency of $180 \mathrm{~Hz}$ and the smallest at 4 and $5 \%$ with the frequency of $250 \mathrm{~Hz}$ when the vibration was applied on the Soleus muscle and tibialis anterior muscle, respectively. The increase rate of muscle force was the greatest at 14 and 13\% with the vibration frequency of $180 \mathrm{~Hz}$ and the smallest at 6 and $6 \%$ with the frequency of $190 \mathrm{~Hz}$ when the vibration was applied on the Lateral Gastrocnemius muscle and Medial Gastrocnemius muscle, respectively. The increase rate of muscle force was the greatest at the frequency of $180 \mathrm{~Hz}$ (Figure 2(b) Lateral Gastrocnemius: $180 \mathrm{~Hz}-190 \mathrm{~Hz} p$ value $=0.007$; Medial Gastrocnemius: $180 \mathrm{~Hz}-190 \mathrm{~Hz} p$ value $=$ $0.019)$.
In Table 2, the increase rate of muscle force showed difference by the intensity of vibration, between the intensity of perception threshold $(100 \%)$ and subthreshold $(80 \%)$ on the Achilles tendon to stimulate triceps surae and tibialis anterior muscle but the difference was not statistically significant.

In Table 3, the increase rate of muscle force showed difference by the intensity of vibration, between the intensity of perception threshold (100\%) and subthreshold $(80 \%)$ on the Achilles tendon to stimulate triceps surae and tibialis anterior muscle but the difference was not statistically significant.

3.2. Change in Muscle Reaction Time according to the Characteristics of Vibration. The following is the results of PMT according to the vibration applied to the muscles of the lower leg through the Achilles tendon and the tibialis anterior tendon. Table 4 shows the muscle reaction time [Sec] when the vibration was applied on the Achilles tendon and the tibialis anterior tendon to stimulate triceps surae and tibialis anterior muscle. The increase rate of muscle force was obtained by comparing the PMT data without stimulation.

Table 4 showed that the muscle reaction time decreased with the vibration stimulation for both the triceps surae and tibialis anterior muscle compared with those without stimulation. The muscle reaction time of both triceps surae and tibialis anterior muscle significantly decreased with the vibration applied on the Achilles tendon (Soleus $p$ value $=0.005$, Lateral Gastrocnemius $p$ value $=0.009$, Medial Gastrocnemius $p$ value $=0.005$, and Tibialis Anterior $p$ value 
TABLE 2: The increase rate of muscle force according to the intensity of vibration, perception threshold (100\%) and subthreshold (80\%) on the Achilles tendon [\%].

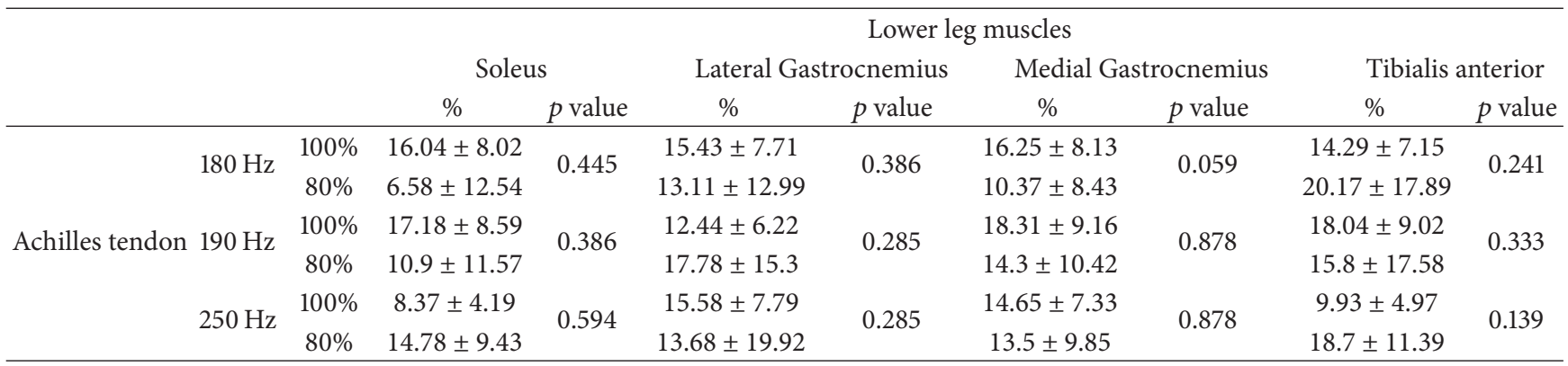

TABLE 3: The increase rate of muscle force according to the intensity of vibration, perception threshold (100\%) and subthreshold (80\%) on the tibialis anterior tendon [\%].

\begin{tabular}{|c|c|c|c|c|c|c|c|c|c|c|}
\hline & & & \multicolumn{8}{|c|}{ Lower leg muscles } \\
\hline & & & \multicolumn{2}{|c|}{ Soleus } & \multicolumn{2}{|c|}{ Lateral Gastrocnemius } & \multicolumn{2}{|c|}{ Medial Gastrocnemius } & \multicolumn{2}{|c|}{ Tibialis anterior } \\
\hline & & & $\%$ & $p$ value & $\%$ & $p$ value & $\%$ & $p$ value & $\%$ & $p$ value \\
\hline \multirow{6}{*}{ Tibialis anterior tendon } & \multirow{2}{*}{$180 \mathrm{~Hz}$} & $100 \%$ & $7.14 \pm 3.57$ & \multirow{2}{*}{0.799} & $12.04 \pm 6.02$ & \multirow{2}{*}{0.386} & $12.22 \pm 6.11$ & \multirow{2}{*}{0.575} & $13.22 \pm 6.61$ & \multirow{2}{*}{0.878} \\
\hline & & $80 \%$ & $9.06 \pm 10.75$ & & $9.89 \pm 7.76$ & & $11.06 \pm 7.12$ & & $8.93 \pm 6.52$ & \\
\hline & \multirow{2}{*}{$190 \mathrm{~Hz}$} & $100 \%$ & $11.84 \pm 5.92$ & \multirow{2}{*}{0.575} & $13.06 \pm 6.53$ & \multirow{2}{*}{0.646} & $10.04 \pm 5.02$ & \multirow{2}{*}{0.799} & $12.74 \pm 6.37$ & \multirow{2}{*}{0.959} \\
\hline & & $80 \%$ & $6.23 \pm 13.04$ & & $8.15 \pm 14.61$ & & $5.72 \pm 8.52$ & & $6.28 \pm 5.99$ & \\
\hline & \multirow{2}{*}{$250 \mathrm{~Hz}$} & $100 \%$ & $12.48 \pm 6.24$ & \multirow{2}{*}{0.508} & $13.68 \pm 6.84$ & \multirow{2}{*}{0.386} & $10.11 \pm 5.06$ & \multirow{2}{*}{0.386} & $9.18 \pm 4.59$ & \multirow{2}{*}{0.114} \\
\hline & & $80 \%$ & $5.82 \pm 20.19$ & & $15.18 \pm 19.52$ & & $7.63 \pm 8.24$ & & $3.11 \pm 6.86$ & \\
\hline
\end{tabular}

TABLE 4: The muscle reaction time by the vibration stimulation applied to the Achilles tendon and the tibialis anterior tendon [Sec].

\begin{tabular}{lcccc}
\hline & Soleus & Lateral Gastrocnemius & Medial Gastrocnemius & Tibialis anterior \\
\hline Nonstimulation & $0.285^{*} \pm 0.060$ & $0.281^{*} \pm 0.060$ & $0.262^{*} \pm 0.059$ & $0.193^{*} \pm 0.072$ \\
Achilles tendon stimulation & $0.199^{*} \pm 0.069$ & $0.203^{*} \pm 0.072$ & $0.188^{*} \pm 0.071$ & $0.124^{*} \pm 0.069$ \\
Tibialis anterior tendon stimulation & $0.196^{*} \pm 0.081$ & $0.198^{*} \pm 0.087$ & $0.187^{*} \pm 0.087$ & $0.125^{*} \pm 0.079$ \\
\hline
\end{tabular}

${ }^{*} p<0.05$. Nonstimulation versus stimulation.

$=0.009)$. The muscle reaction time of both triceps surae and tibialis anterior muscle significantly decreased with the vibration applied on the tibialis anterior tendon (Soleus $p$ value $=0.005$, Lateral Gastrocnemius $p$ value $=0.013$, Medial Gastrocnemius $p$ value $=0.005$, and tibialis anterior $p$ value $=0.012$ ).

Figures 3(a) and 3(b) show the muscle reaction time [Sec] according to the vibration frequency applied on the Achilles tendon and tibialis anterior tendon. The muscle reaction time was significantly shorter with the vibration stimulation on the Achilles tendon at $180 \mathrm{~Hz}$ frequency compared with that without stimulation (Figure 3(a)). Moreover, the muscle reaction time at the frequency of $180 \mathrm{~Hz}$ was the shortest compared with the other frequencies.

In Table 5, the reaction time showed difference by the intensity of vibration, the intensity of perception threshold (100\%) and subthreshold (80\%) on the Achilles tendon to stimulate triceps surae and tibialis anterior muscle, but the difference was not statistically significant.

In Table 6 , the reaction time showed difference by the intensity of vibration, the intensity of perception threshold $(100 \%)$ and subthreshold $(80 \%)$ on the Achilles tendon to stimulate triceps surae and tibialis anterior muscle, but the difference was not statistically significant.

\section{Discussion}

4.1. Change of Muscle Force according to the Characteristics of Vibration. This study analyzed the change of muscle force according to the characteristics of vibration using MVC. The muscle force significantly increased with the vibration stimulation compared to that without any stimulation. This result could be assumed that the vibration applied on the Achilles tendon and tibialis anterior tendon improved the muscle force of the triceps surae and tibialis anterior muscle which, in turn, improve the stability of the lower leg during gait. The vibration stimulation applied on the Achilles tendon rather than the tibialis anterior tendon proved to be more effective, which meant the vibration applied to the Achilles tendon had a greater effect on the muscle force increase. A vibration stimulation with different vibration frequency on the Achilles tendon and tibialis anterior tendon was applied. The results showed that the vibration frequency of $180 \mathrm{~Hz}$ had the greatest increase of muscle force for both 


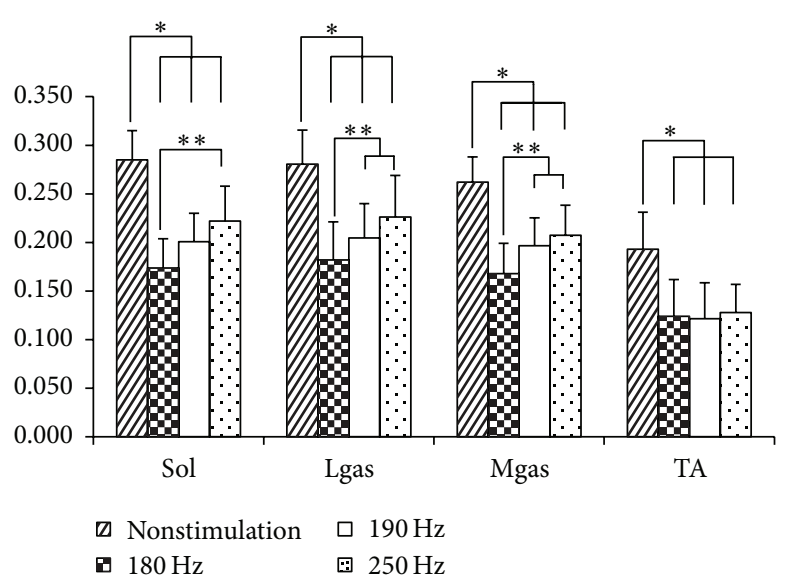

(a)

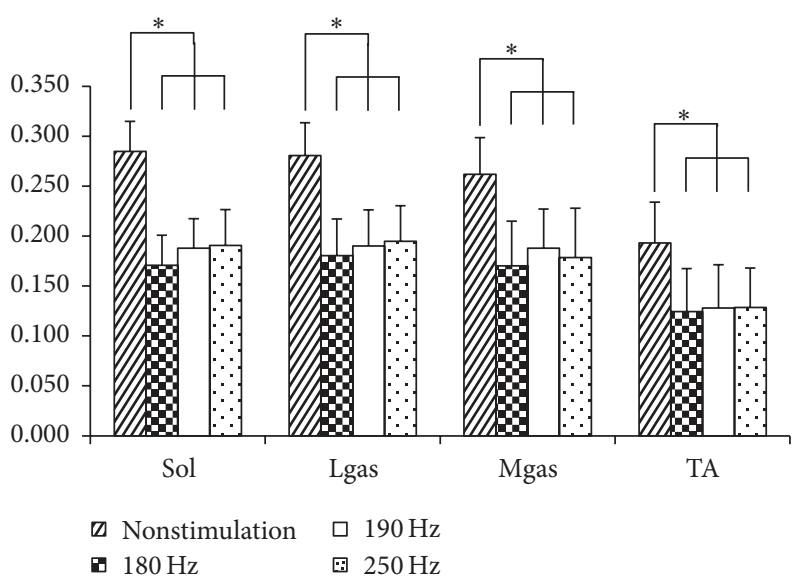

(b)

Figure 3: The muscle reaction time by the vibration stimulation applied on the Achilles tendon and tibialis anterior tendon according to vibration frequency $[\mathrm{Sec}]\left({ }^{*} p<0.05\right.$. nonstimulation versus other frequencies, ${ }^{* *} p<0.05 .180 \mathrm{~Hz}$ versus other frequencies). (a) Achilles tendon. (b) Tibialis anterior tendon.

TABLE 5: The muscle reaction time according to the intensity of vibration, perception threshold (100\%) and subthreshold (80\%) on the Achilles tendon $[\mathrm{Sec}]$.

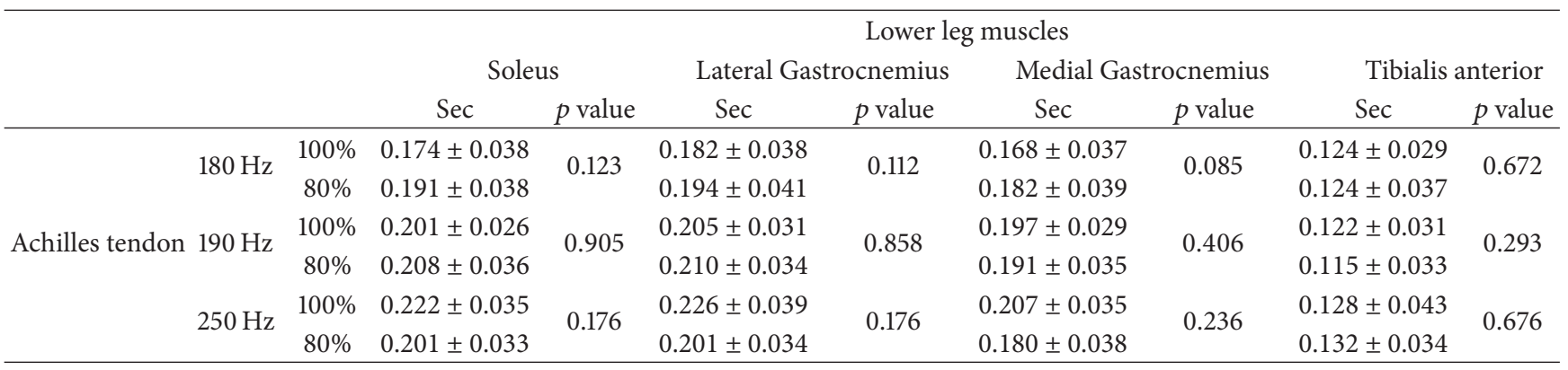

the Achilles tendon and tibialis anterior tendon. It could be assumed that the change of muscle force is dependent on the frequency from this result. The vibration stimulation with the intensity of perception threshold and subthreshold (80\%) was applied on the Achilles tendon and tibialis anterior tendon. The results showed that the increase rate of muscle force was different from the vibration intensity when the vibration stimulation was applied on the triceps surae and tibialis anterior muscle via the Achilles tendon. The increase rate of muscle force was also different from the vibration intensity when the vibration stimulation was applied on the triceps surae and tibialis anterior muscle via the tibialis anterior tendon. However, the difference was not statistically significant for both the Achilles tendon and tibialis anterior tendon stimulation. This result means that the vibration with the intensity of perception threshold and subthreshold affects the muscle force at the same extent.

4.2. Change in Muscle Reaction Time according to the Characteristics of Vibration. The change on the muscle reaction time according to the characteristics of vibration was analyzed using PMT. The muscle reaction time significantly decreased with the vibration stimulation compared to that without any stimulation. From this result, the vibration stimulation applied to the muscles of the lower leg shortened the muscle reaction time from the sensory system, CNS to the muscular system. In other words, this result could be assumed that the efficiency in transmitting/processing nerve impulses improved. A vibration stimulation with different vibration frequency on the Achilles tendon and tibialis anterior tendon was applied. The results showed that the muscle reaction time was significantly shorter at the frequency of $180 \mathrm{~Hz}$ for both the Achilles tendon and tibialis anterior tendon stimulation compared to those of other frequencies. It means that the reaction time is dependent on the vibration frequency. The vibration stimulation with the intensity of perception threshold and subthreshold (80\%) was applied on the Achilles tendon and tibialis anterior tendon. The results showed that the muscle reaction time was different from the intensity but statistically insignificant. The results mean that the vibration with the intensity of perception threshold and subthreshold equally affects the muscle reaction time.

\section{Conclusion}

This study investigated the effect of mechanical vibration stimulation applied on the Achilles tendon and tibialis anterior tendon according to the characteristics of vibration 
TABLE 6: The muscle reaction time according to the intensity of vibration, perception threshold (100\%) and subthreshold (80\%) on the tibialis anterior tendon $[\mathrm{Sec}]$.

\begin{tabular}{|c|c|c|c|c|c|c|c|c|c|c|}
\hline & & & \multicolumn{8}{|c|}{ Lower leg muscles } \\
\hline & & & \multicolumn{2}{|c|}{ Soleus } & \multicolumn{2}{|c|}{ Lateral Gastrocnemius } & \multicolumn{2}{|c|}{ Medial Gastrocnemius } & \multicolumn{2}{|c|}{ Tibialis anterior } \\
\hline & & & Sec & $p$ value & Sec & $p$ value & $\mathrm{Sec}$ & $p$ value & $\mathrm{sec}$ & $p$ value \\
\hline \multirow{6}{*}{$\begin{array}{l}\text { Tibialis } \\
\text { anterior } \\
\text { tendon }\end{array}$} & \multirow{2}{*}{$180 \mathrm{~Hz}$} & $100 \%$ & $0.171 \pm 0.033$ & \multirow{2}{*}{0.859} & $0.181 \pm 0.036$ & \multirow{2}{*}{0.574} & $0.170 \pm 0.036$ & \multirow{2}{*}{0.944} & $0.124 \pm 0.036$ & \multirow{2}{*}{0.071} \\
\hline & & $80 \%$ & $0.204 \pm 0.051$ & & $0.203 \pm 0.052$ & & $0.189 \pm 0.054$ & & $0.107 \pm 0.028$ & \\
\hline & \multirow{2}{*}{$190 \mathrm{~Hz}$} & $100 \%$ & $0.188 \pm 0.037$ & \multirow{2}{*}{0.549} & $0.190 \pm 0.045$ & \multirow{2}{*}{0.513} & $0.188 \pm 0.039$ & \multirow{2}{*}{0.959} & $0.128 \pm 0.049$ & \multirow{2}{*}{0.302} \\
\hline & & $80 \%$ & $0.191 \pm 0.043$ & & $0.197 \pm 0.046$ & & $0.191 \pm 0.044$ & & $0.118 \pm 0.038$ & \\
\hline & \multirow{2}{*}{$250 \mathrm{~Hz}$} & $100 \%$ & $0.191 \pm 0.041$ & \multirow{2}{*}{0.514} & $0.195 \pm 0.043$ & \multirow{2}{*}{0.476} & $0.178 \pm 0.043$ & \multirow[t]{2}{*}{0.906} & $0.128 \pm 0.040$ & \multirow{2}{*}{0.291} \\
\hline & & $80 \%$ & $0.203 \pm 0.039$ & & $0.207 \pm 0.039$ & & $0.188 \pm 0.045$ & & $0.143 \pm 0.047$ & \\
\hline
\end{tabular}

including the presence of vibration, frequency, and intensity on the muscles of the lower leg and the following results were obtained. The change of muscle force and muscle reaction time according to the characteristics of vibration showed that both improved with the vibration stimulation compared to those without any stimulation. The frequency of $180 \mathrm{~Hz}$ showed the best results and the changes of muscle force and reaction time were dependent on the vibration frequency. The effect of vibration with the intensity of perception threshold and subthreshold on the muscle force and muscle reaction time was the same. In conclusion, a local tendon stimulation using vibration of $180 \mathrm{~Hz}$ frequency and perception subthreshold intensity would improve the muscle force and muscle reaction time and contribute the stability of the lower leg during gait.

\section{Competing Interests}

The authors declare that there is no conflict of interests regarding the publication of this paper.

\section{Acknowledgments}

This work was supported by the Research Foundation of Korea (NRF) grant funded by the Korea government (MSIP) (NRF-2014R1A2A1A11053073) and research funds of Chonbuk National University in 2015.

\section{References}

[1] M. E. Tinetti, S. K. Inouye, T. M. Gill, and J. T. Doucette, "Shared risk factors for falls, incontinence, and functional dependence: unifying the approach to geriatric syndromes," The Journal of the American Medical Association, vol. 273, no. 17, pp. 1348-1353, 1995.

[2] J. P. Perry, Gait Analysis: Normal and Pathological Function, SIACK, Downey, Calif, USA, 1992.

[3] J. O. Judge, "Balance training to maintain mobility and prevent disability," American Journal of Preventive Medicine, vol. 25, no. 3, pp. 150-156, 2003.

[4] D. M. Buchner, E. B. Larson, E. H. Wagner, T. D. Koepsell, and B. J. De Lateur, "Evidence for a non-linear relationship between leg strength and gait speed," Age and Ageing, vol. 25, no. 5, pp. 386-391, 1996.

[5] H. Issever, C. Aksoy, H. Sabuncu, and A. Karan, "Vibration and its effects on the body," Medical Principles and Practice, vol. 12, no. 1, pp. 34-38, 2003.

[6] H. J. So, K. Y. Kim, S. H. Kim, N. G. Kim, and D. W. Kim, "The Analysis of GRF and joint angles of young and older adult by vibration stimulation on the ankle-joint in stair-descent activity," Journal of Biomedical Engineering Research, vol. 32, no. 1, pp. 61-73, 2011.

[7] K. Kwak, H. So, S. Kim, Y. Yang, N. Kim, and D. Kim, "Effects of somatosensory stimulation on lower-limb joint kinetic of older adult during stair descent," Journal of Biomedical Engineering Research, vol. 32, no. 2, pp. 93-104, 2011.

[8] D. C. Gravelle, C. A. Laughton, N. T. Dhruv et al., "Noiseenhanced balance control in older adults," NeuroReport, vol. 13, no. 15, pp. 1853-1856, 2002.

[9] R. Dickstein, Y. Laufer, and M. Katz, "TENS to the posterior aspect of the legs decreases postural sway during stance," Neuroscience Letters, vol. 393, no. 1, pp. 51-55, 2006.

[10] G. Eklund, "General features of vibration-induced effects on balance," Upsala Journal of Medical Sciences, vol. 77, no. 2, pp. 112-124, 1972.

[11] E. L. Curry and J. A. Clelland, "Effects of the asymmetric tonic neck reflex and high-frequency muscle vibration on isometric wrist extension strength in normal adults," Physical Therapy, vol. 61, no. 4, pp. 487-495, 1981.

[12] S. R. Kang, G. Y. Jeong, J. J. Bae et al., "Effect of muscle function and muscular reaction of knee joint in the twenties on the whole body vibration exercise," Journal of the Korean Society for Precision Engineering, vol. 30, no. 7, pp. 762-768, 2013.

[13] O. Handy and M. Volker, Vibration Thresholds and Equal Vibration Levels at the Human Fingertip and Palm, ICA, Kyoto, Japan, 2004.

[14] A. Picu, "Study about evaluation of human exposure to handtransmitted vibration," Journal of Science and Arts, vol. 2, pp. 355-360, 2010.

[15] T. Sakurai, M. Konyo, S. Okamoto, and S. Tadokoro, "Research of conditions of stimulus for inducing grasping force control reflex," in Proceedings of the IEEE/SICE International Symposium on System Integration (SII '10), pp. 408-413, Sendai, Japan, December 2010.

[16] K. Kwak, H. Kim, and D. Kim, "Variation of ankle biomechanical property according to vibro-perception threshold and 
vibration frequency," Biomedical Engineering Letters, vol. 6, no. 1, pp. 16-25, 2016.

[17] J.-W. Kim, H.-Y. Jeong, H.-H. Kim et al., "Comparison of reaction times of ankle joint muscles in the elderly men and women," Journal of Biomedical Engineering Research, vol. 33, no. 4, pp. 163-168, 2012. 


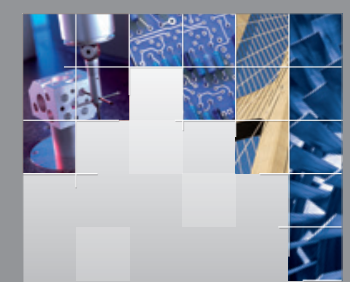

\section{Enfincering}
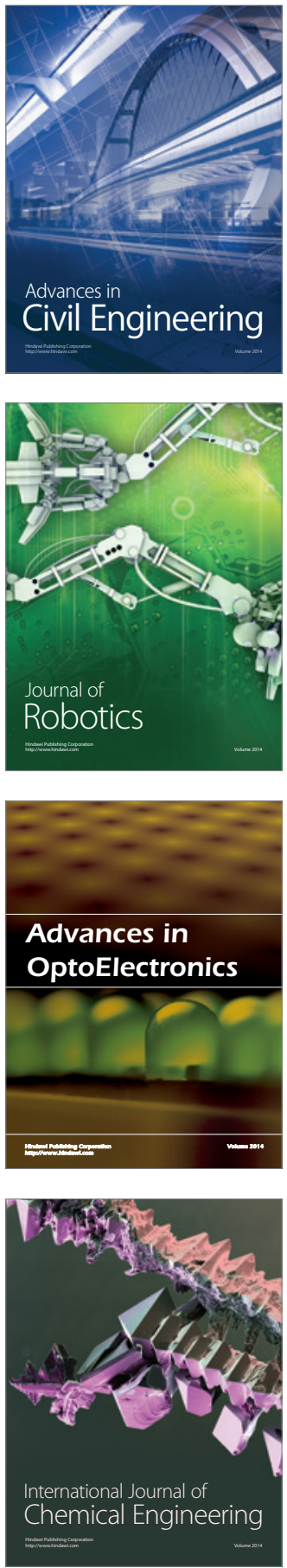

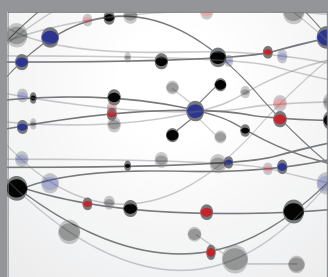

The Scientific World Journal

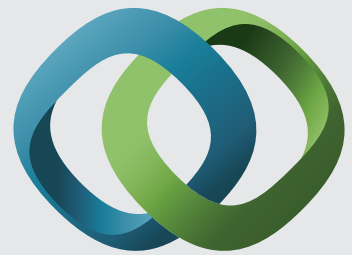

\section{Hindawi}

Submit your manuscripts at

http://www.hindawi.com
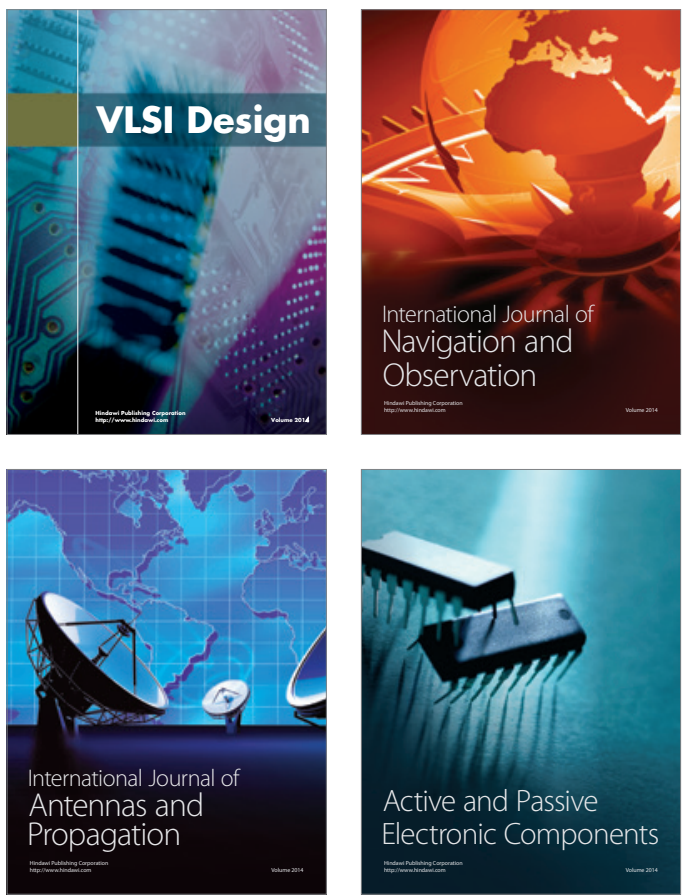
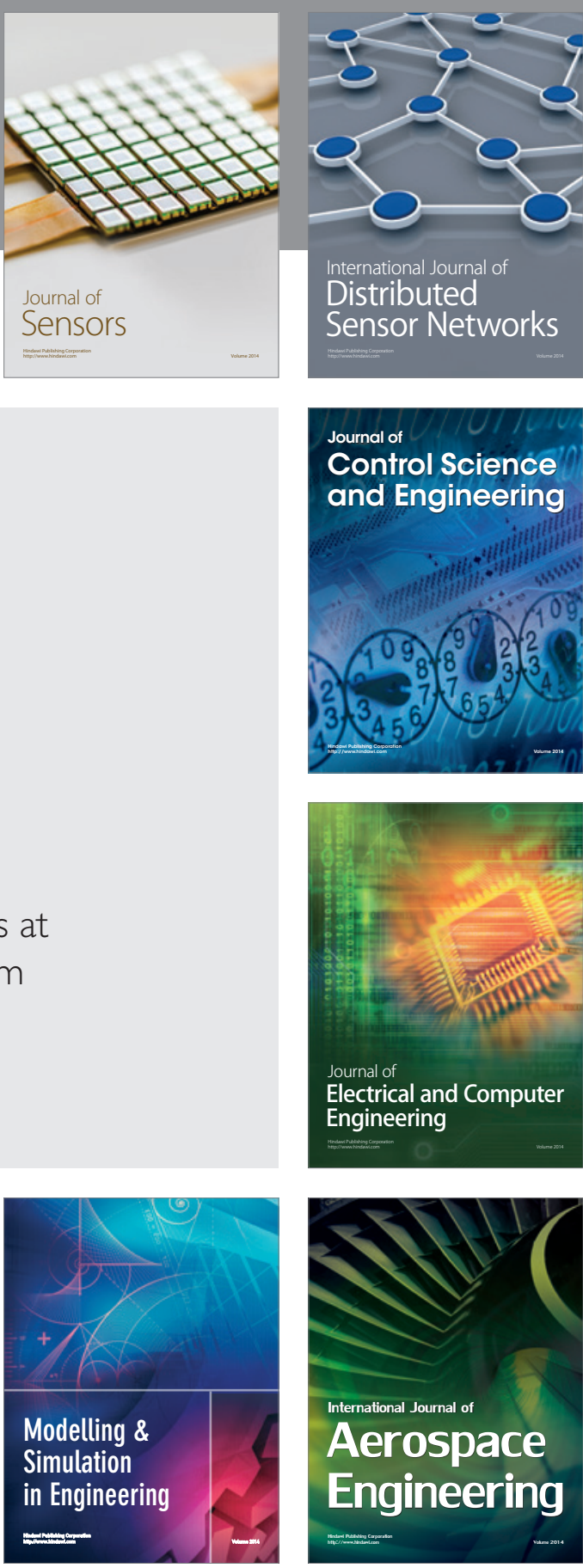

International Journal of

Distributed

Sensor Networks

Journal of

Control Science

and Engineering
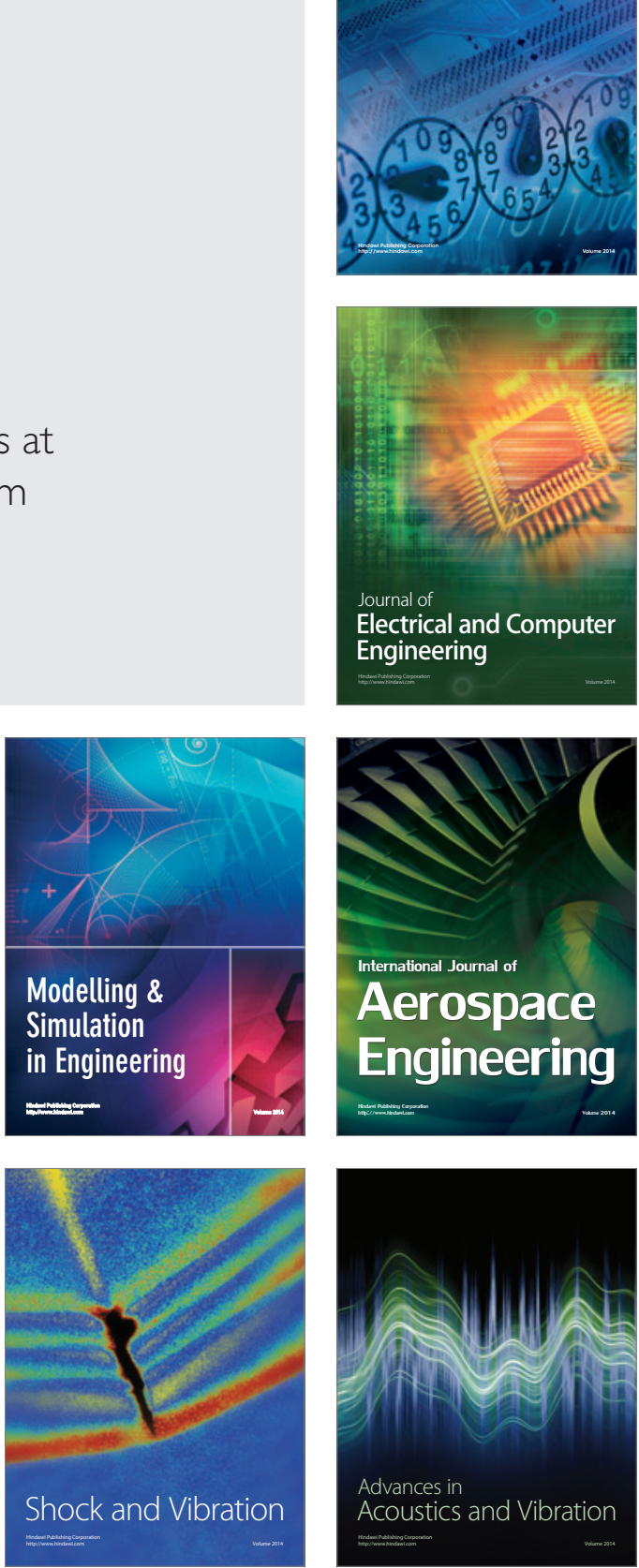